\title{
Current status of diagnosis and treatment of advanced non-small cell lung cancer in China during the COVID-19 pandemic
}

\author{
Ming Liu ${ }^{1}$, Wei Zhao ${ }^{2}$, Shiyue $\mathrm{Li}^{1}$, Liangan Chen ${ }^{2}$, Chengzhi Zhou ${ }^{1 \wedge}$ \\ ${ }^{1}$ National Clinical Research Center for Respiratory Disease, State Key Laboratory of Respiratory Disease, Guangzhou Institute of Respiratory \\ Health, The First Affiliated Hospital of Guangzhou Medical University, Guangzhou, China; ${ }^{2}$ Department of Pulmonary \& Critical Care Medicine, \\ Chinese PLA General Hospital, Beijing, China \\ Contributions: (I) Conception and design: M Liu; (II) Administrative support: W Zhao; (III) Provision of study materials or patients: S Li; (IV) \\ Collection and assembly of data: L Chen; (V) Data analysis and interpretation: C Zhou; (VI) Manuscript writing: All authors; (VII) Final approval of \\ manuscript: All authors. \\ Correspondence to: Chengzhi Zhou. National Clinical Research Center for Respiratory Disease, State Key Laboratory of Respiratory Disease, \\ Guangzhou Institute of Respiratory Health, The First Affiliated Hospital of Guangzhou Medical University, No. 151 Yanjiang Road, Guangzhou \\ 510120, China. Email: doctorzcz@163.com; Liangan Chen. Department of Pulmonary \& Critical Care Medicine, Chinese PLA General Hospital, \\ Beijing, China. Email: chenliangan301@163.com.
}

Background: The purpose of this study was to investigate the current status of diagnosis and treatment of Chinese patients with advanced non-small cell lung cancer (NSCLC) and the expert recommendation of the therapeutic regimens during the coronavirus disease 2019 (COVID-19) pandemic, and to analyze and summarize relevant rules.

Methods: Under the planning and coordination of the Lung Cancer Group of the Chinese Thoracic Society, we performed an online questionnaire survey among experts in lung cancer and patients with NSCLC. Convenience sampling was adopted for questionnaire survey of experts, and random sampling was adopted for questionnaire survey of patients. After collecting and sorting out the questionnaire, a descriptive analysis of the data was performed.

Results: Within $24 \mathrm{~h}$ from the distribution of questionnaires to the deadline, a total of 808 and 321 valid questionnaires from patients and experts were collected across China, respectively. There were $83.81 \%$ of the experts performed moderately and strongly anti-tumor therapy for patients with NSCLC during the COVID-19 pandemic. $76.6 \%$ of patients mainly receive online public welfare treatment, and the patient satisfaction rate reached up to $64.97 \%$. For driver gene-positive patients with advanced NSCLC and nonCOVID-19, $82.87 \%$ of the experts recommended first-line simple targeted therapy, and $12.77 \%$ of the experts recommended targeted therapy with oral anti-angiogenic drugs. For patients who were unable to return to the hospital for treatment and showed resistance to the tyrosine kinase inhibitor (TKI) therapy, $92.21 \%$ of the experts recommended oral anti-angiogenic drugs as the third-line home-based therapy and above. For patients with advanced NSCLC combined with COVID-19, $98.76 \%$ and $95.95 \%$ of the experts recommended discontinuation of chemotherapy and immunotherapy, respectively.

Conclusions: During COVID-19, most Chinese patients with NSCLC were still able to receive timely diagnosis and treatment either by online public welfare consultation or at nearby hospitals.

Keywords: Coronavirus disease 2019 (COVID-19); advanced non-small cell lung cancer (NSCLC); treatment; diagnosis; drug

\footnotetext{
$\wedge$ ORCID: 0000-0001-5935-184X.
} 
Submitted Jan 11, 2021. Accepted for publication Aug 30, 2021.

doi: 10.21037/apm-21-72

View this article at: https://dx.doi.org/10.21037/apm-21-72

\section{Introduction}

In 2020, coronavirus disease 2019 (COVID-19) has spread to over 200 countries and districts throughout the world, causing enormous economic and social losses. China is rapidly recovering from this public health emergency. Chinese experiences in fighting COVID-19 may provide a reference for other countries in the throes of COVID-19. COVID-19 is mainly spread by droplet transmission and contact transmission with extremely high infectivity, which results in continuous human-to-human transmission (1).

An analysis on 72,314 patients with COVID-19 from Chinese mainland showed that the crude mortality rate of COVID-19 overall patient population was $2.3 \%$. The crude mortality rate of the patients with COVID-19 combined with a history of malignancies was increased to $5.6 \%$ (2). Another study on 1,590 patients with COVID-19 indicated that the patients combined with malignancies had a higher risk for the novel emerged coronavirus (SARS-CoV-2) infection and worse prognosis (3). Among 18 patients with COVID-19 and different malignancies, lung cancer (5/18, $28 \%$ ) was the most common cancer (3).

It is of high practical importance to understand the current status of the treatment of Chinese patients with non-small cell lung cancer (NSCLC) during the COVID-19 pandemic. Therefore, we performed an online survey to understand the current status of diagnosis and treatment of Chinese patients with NSCLC and recommendations of therapeutic regimens of experts in NSCLC during the COVID-19 pandemic. We hope that our study may be of some use for international peer experts and patients during the COVID-19 pandemic. We present the following article in accordance with the SURGE reporting checklist (available at https://apm.amegroups.com/article/ view/10.21037/apm-21-72/rc).

\section{Methods}

\section{Subjects}

The study was conducted in accordance with the Declaration of Helsinki (as revised in 2013). The study was approved by the ethics committee of The First Affiliated Hospital of Guangzhou Medical University (Ethics
Number: 2020 No. 35). All subjects signed informed consent. Under the planning and coordination of the Lung Cancer Group of the Chinese Thoracic Society, 330 experts in lung cancer and 820 patients with NSCLC across China received an online questionnaire survey. A total of 321 valid questionnaires were collected from the experts in the Lung Cancer Group of the Chinese Thoracic Society, Chinese Society of Clinical Oncology (CSCO) Young Lung Cancer Group, and China Respiratory Oncology Collaboration (CROC). Meanwhile, 808 valid questionnaires were collected from Chinese patients with NSCLC. The effective response rate of the questionnaire was $98.17 \%$. The surveyed experts came from the department of respiratory medicine, department of oncology, department of thoracic surgery, and department of radiotherapy.

\section{Questionnaire survey}

Under the planning and coordination by the Lung Cancer Group of the Chinese Thoracic Society, 16 problems related to the diagnosis and treatment of lung cancer that were of concern to experts and have clinical significance were included in the questionnaire. The consultation and answer part were additionally designed for the questionnaire survey to the patients to ensure the accuracy of the information collected. The questionnaire to expert contained 36 problems related to the treatment of advanced NSCLC. A total of 321 experts with experience received the questionnaire survey.

Convenience sampling was adopted for questionnaire survey of experts, and random sampling was adopted for questionnaire survey of patients. The questionnaire for experts was designed with single-choice questions, and the questionnaire for patients was designed with both singlechoice and multiple-choice questions. This article does not contain any studies with human participants or animals performed by any of the authors.

\section{Statistical analysis}

Statistical analysis was performed using the Statistical Package for Social Science (SPSS) software, version 22.0 software. Categorical variables were described by 
Table 1 Basic information of patients

\begin{tabular}{|c|c|}
\hline Items & Proportion, \% \\
\hline \multicolumn{2}{|l|}{ Gender } \\
\hline Male & 68.56 \\
\hline Female & 31.44 \\
\hline \multicolumn{2}{|l|}{ Ages (years) } \\
\hline$<40$ & 11.30 \\
\hline $40-59$ & 41.37 \\
\hline $60-69$ & 31.06 \\
\hline$\geq 70$ & 16.27 \\
\hline \multicolumn{2}{|l|}{ Case types } \\
\hline NSCLC & 75.49 \\
\hline SCLC & 17.45 \\
\hline Others & 7.05 \\
\hline \multicolumn{2}{|l|}{ Driving gene types } \\
\hline Positive & 63.24 \\
\hline Negative & 36.76 \\
\hline \multicolumn{2}{|l|}{ PD-L1 examination } \\
\hline No & 73.02 \\
\hline Yes, PD-L1 <1\% & 18.19 \\
\hline Yes, $1 \%<$ PD-L1 < $49 \%$ & 7.18 \\
\hline Yes, PD-L1 >50\% & 1.61 \\
\hline \multicolumn{2}{|l|}{ Contact history of COVID-19 } \\
\hline No & 97.77 \\
\hline $\begin{array}{l}\text { Yes, but the infection was basically eliminated } \\
\text { after the isolation period }\end{array}$ & 1.61 \\
\hline Yes, but it has been cured & 0.62 \\
\hline
\end{tabular}

NSCLC, non-small cell lung cancer; SCLC, small cell lung cancer; PD-L1, programmed death ligand-1; COVID-19, coronavirus disease 2019.

frequencies and percentages. Measurement data were expressed as means $\pm \mathrm{SD}$, and compared by ANOVA. $\mathrm{P}<0.05$ was considered as statistical different.

\section{Results}

\section{Baseline information}

A total of 808 valid questionnaires from patients and 321 valid questionnaires from experts were collected all across
Table 2 Basic information of experts

\begin{tabular}{lc}
\hline Items & Proportion, $\mathrm{n}(\%)$ \\
\hline Distribution of provinces and cities & $71(22.12)$ \\
Guangdong Province & $48(14.95)$ \\
Henan Province & $42(13.08)$ \\
Beijing City & $21(6.54)$ \\
Zhejiang Province & \\
Distribution of hospital & $287(89.41)$ \\
Grade A of level III hospital & $34(10.59)$ \\
Under grade A of level III hospital & \\
Distribution of department & $157(48.91)$ \\
Distribution of respiratory medicine & $131(40.81)$ \\
Oncology department & $15(4.67)$ \\
Distribution of thoracic surgery & $14(4.36)$ \\
Distribution of radiotherapy department & $192(59.81)$ \\
Titles & \\
Attending doctor & $75(23.36)$ \\
Associate senior doctor and above & $246(76.64)$ \\
Diagnosis and treatment of CovID-19 & \\
Direct participation & \\
\hline COVID-19, coronavirus disease 2019. & \\
\hline
\end{tabular}

China. The baseline information of patients and experts were shown in Tables 1,2. There were $68.56 \%$ males and $31.44 \%$ females. For age distribution, $11.3 \%$ patients were aged below 40 years old, $41.37 \%$ were $40-59$ years old, and $31.0 \%$ were $60-69$ years old. Among all patients, $75.49 \%$ patients had NSCLC, $17.45 \%$ had SCLC, and $7.05 \%$ patients had other pathological types. Moreover, $63.24 \%$ patients were positive for the driver gene, and $36.76 \%$ were negative for the driver gene. Programmed death ligand-1 (PD-L1) test was not performed in $73.02 \%$ patients. Moreover, $18.19 \%$ patients were PD-L1 of $<1 \%, 7.18 \%$ were PD-L1 of $1-49 \%$, and $1.16 \%$ were PD-L1 of $>50 \%$. For the history of COVID-19 exposure, $97.77 \%$ had no history of COVID-19 exposure, $1.61 \%$ had the history of COVID-19 exposure in which the possibility of infection was excluded after 14 days of quarantine, and $0.62 \%$ were once infected by COVID-19 and already cured.

A total of 321 experts surveyed came from 28 provinces 


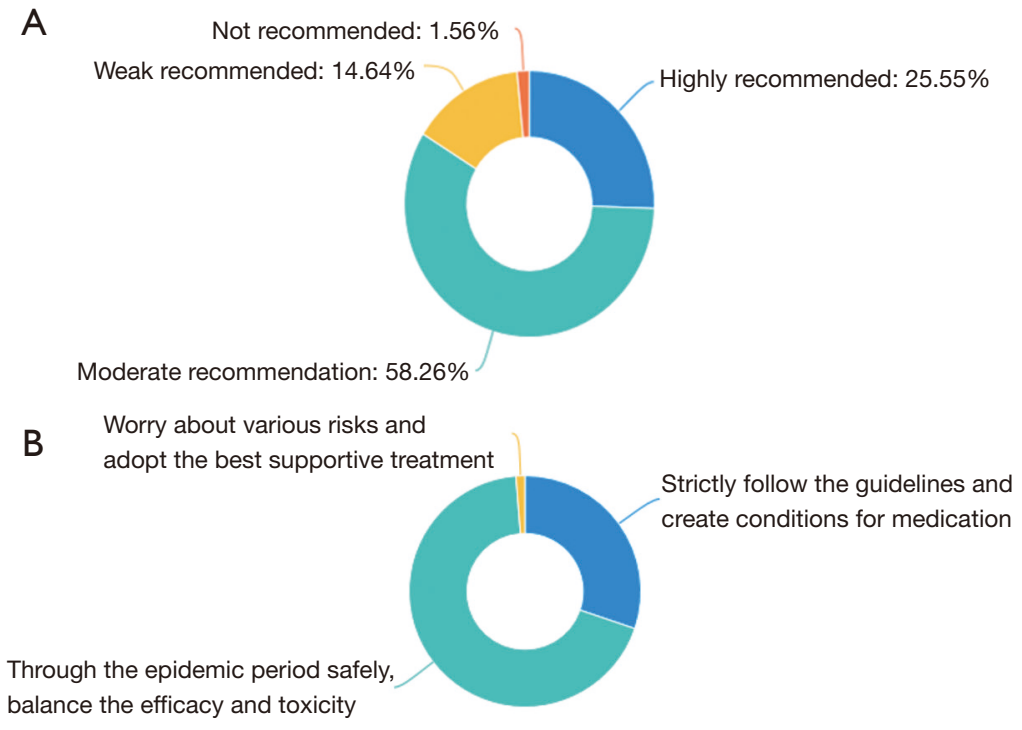

Figure 1 Expert advice on diagnosis and treatment of advanced NSCLC during the COVID-19 pandemic. (A) Treatment; (B) medication. NSCLC, non-small cell lung cancer; COVID-19, coronavirus disease 2019.

in China, including 71 from Guangdong Province (22.12\%), 48 from Henan Province 48 (14.95\%), 42(13.08\%) from Beijing, and 21 (6.54\%) from Zhejiang Province. Among them, 157 experts $(48.91 \%)$ surveyed came from the department of respiratory medicine, 131 (40.81\%) from the department of oncology, $15(4.67 \%)$ from the department of thoracic survey, and 14 (4.36\%) from the department of radiotherapy. A total of 192 experts $(59.81 \%)$ participated in the diagnosis and treatment of COVID-19.

\section{The diagnosis and treatment of advanced NSCLC during the COVID-19 pandemic}

For the therapeutic principle, during the COVID-19 pandemic, $83.81 \%$ of the experts moderately and strongly recommended anti-tumor therapy for the patients with advanced NSCLC, while $14.64 \%$ only made a weak recommendation of the anti-tumor treatment. A total of $68.54 \%$ experts recommended waiting for the end of the pandemic and weighing the trade-offs between efficacy and toxicity, $30.22 \%$ experts recommended patients to strictly follow the guidelines and create favorable conditions for anti-tumor medication, and 4 (1.25\%) worried about all kinds of risks and only recommended supportive treatment. The results were shown in Figure 1.

For the access pathway to health care, during the
COVID-19 pandemic, patients received primary diagnosis and treatment via online public welfare consultation, including telephone of $39.23 \%$, WeChat of $22.15 \%$ and gratuitous online treatment of $15.22 \%$, accounting for $76.6 \%$. Only $5.69 \%$ of the patients paid for the online medical consultation. The satisfaction rating of patients who received online diagnosis and prescription reached $64.97 \%$. Moreover, 249 experts (77.57\%) believed that online diagnosis and prescription had limited effect on patients and could solve limited problems. However, 72 experts $(22.43 \%)$ believed that online diagnosis and prescription could solve most of the problems and were worthy of popularization.

In the choice of hospitals, before or during the pandemic, local first-class hospitals at grade 3 were the preferred choice for patients $(53.59 \%$ or $43.19 \%)$. During the pandemic, the percentage of patients who sought medical care at nearby hospitals (non-first-class hospitals at grade 3) was increased from $17.45 \%$ to $35.02 \%$, the percentage of patients who sought medical care at local renown firstclass hospitals at grade 3 was decreased from $21.53 \%$ to $9.41 \%$. The percentage of patients who sought medical care at domestic renown first-class hospitals at grade 3 was decreased from $5.82 \%$ to $1.98 \%$.

During the pandemic, the reasons for changing the therapeutic regimens among patients with NSCLC were as follows: doctor's recommendation for $49.38 \%$, postponing 
of the plan for doctor visits for $46.66 \%$, and difficulty in booking hospital beds for $38 \%$. There were $56.07 \%$ experts who recommended patients to seek medical care nearby, $31.78 \%$ recommended patients to make a decision after online expert consultation, and $10.59 \%$ recommended patients to seek treatment at the original healthcare institutes.

\section{Changes in the medication regimens for patients with advanced NSCLC and expert recommendations}

The percentage of patients receiving targeted therapy was increased from $24.26 \%$ to $35.27 \%$ during the pandemic. The percentage of patients receiving multi-target antiangiogenic drugs was increased from $10.15 \%$ to $21.66 \%$. The percentage of patients receiving chemotherapy and combination chemotherapy was decreased from $36.26 \%$ to $15.97 \%$. The percentage of patients receiving immunotherapy and combination immunotherapy was decreased from $3.84 \%$ to $2.6 \%$.

Among various medication options for advanced NSCLC during the pandemic, 270 experts $(84.11 \%)$ recommended the use of targeted therapy, 34 (10.59\%) recommended oral anti-angiogenic drugs, 11 (3.43\%) recommended immunotherapy, and $6(1.87 \%)$ recommended chemotherapy. However, 156 experts (48.6\%) did not recommend immunotherapy, 153 (47.66\%) did not recommend chemotherapy, 5 (1.56\%) did not recommend targeted therapy, 5 (1.56\%) did not recommend the intravenous anti-angiogenic drugs, and $2(0.62 \%)$ did not recommend the intravenous anti-angiogenic drugs.

\section{Expert recommendations on medication regimens for patients with advanced NSCLC and non-COVID-19}

For patients with advanced NSCLC without COVID-19, it is very important to treat timely when necessary, and to distinguish the adverse events and symptoms related to COVID-19.

(I) For patients with driver gene-positive advanced NSCLC, 266 experts $(82.87 \%)$ recommended simple epidermal growth factor receptor-tyrosine kinase inhibitor (EGFR-TKI) therapy as the firstline therapy, 41 (12.77\%) recommended EGFRTKI therapy combined with anti-angiogenic drugs, but EGFR-TKI with chemotherapy should be used with caution. During the pandemic,
$35.27 \%$ patients chose the targeted therapy, while only $8.79 \%$ chose the combination targeted therapy. This phenomenon agreed with the expert recommendation. EGFR-TKIs have a wellunderstood mechanism and explicit effect, and they are suitable for home-based treatment, thereby avoiding the risk of COVID-19 infection caused by EGFR-TKI combined with other treatment.

(II) For driver gene-negative patients with PD-L1 $\geq 50 \%, 198$ experts $(61.68 \%)$ recommended simple immunotherapy, 58 (18.07\%) recommended immunotherapy combined with chemotherapy, 33 $(10.28 \%)$ recommended oral anti-angiogenic drugs combined with chemotherapy.

(III) For the driver gene-negative patients with PDL1 of $1-49 \%$, different therapies were ranked in descending order of recommended favorability. Chemotherapy combined with immunotherapy was $39.25 \%$, simple chemotherapy was $21.18 \%$, and chemotherapy combined with anti-angiogenic drugs was $19.94 \%$. The choice of specific therapeutic regimens for individual patients depended on weighing the trade-offs between efficacy and adverse events of anti-tumor therapy.

(IV) For patients with driver gene negative and PDL1-negative, different therapies were ranked in descending order of recommended favorability. Chemotherapy combined with immunotherapy was $46.42 \%$, simple chemotherapy was $32.09 \%$, and chemotherapy combined with anti-angiogenic drugs was $7.17 \%$. During the pandemic, $15.97 \%$ patients chose chemotherapy with or without other treatments, $7.92 \%$ chose multi-target antiangiogenic drugs. This phenomenon suggested that among the patients with advanced NSCLC who were not suitable for oral targeted drugs, chemotherapy still occupied an important status.

(V) For the driver gene-positive or driver gene-negative patients with advanced NSCLC who were unable to return to hospitals for treatment and showed resistance to the TKI therapy, 296 experts (92.21\%) recommended oral anti-angiogenic drugs as the third-line home-based therapy and above. During the pandemic, $21.66 \%$ patients chose multitargeted anti-angiogenic drugs, $7.92 \%$ chose multitargeted anti-angiogenic drugs combined with other treatments. Multi-targeted anti-angiogenic 
drugs provided another option for patients during the COVID-19 pandemic.

\section{Expert recommendations on medication regimens for patients with advanced NSCLC combined with COVID-19}

First of all, the patients with COVID-19 should be quarantined and receive proper treatment according to the guidelines for the diagnosis and treatment of COVID-19. For driver gene-positive patients with NSCLC and COVID-19, if the condition was mild and ordinary, they should be given targeted therapy continuously (46.11\%), or targeted therapy be discontinued (41.43\%). Moreover, if the condition was severe or critical, the targeted therapy should be discontinued (86.91\%). For patients with advanced NSCLC combined with COVID-19, during the chemotherapy, $98.76 \%$ experts recommended discontinuation of the targeted therapy. If patients were combined with COVID-19 during the immunotherapy, $95.95 \%$ experts recommended discontinuation of the immunotherapy.

After the recovery of advanced NSCLC from COVID-19, the physical conditions of the patients should be comprehensively evaluated. High-efficiency and lowtoxicity medications are recommended, and the anti-tumor treatment should be postponed. Targeted therapy should be administered after the patients were discharged from the hospital for COVID-19 for over 2 weeks. Chemotherapy, immunotherapy and intravenous anti-angiogenic drugs should be given at over four weeks after the patients were discharged from the hospital for COVID-19.

\section{Discussion}

Our survey was the first one on the current status of treatment among patients with advanced NSCLC and the expert recommendations on therapeutic regimens for these patients. The patients surveyed came from over 20 provinces in China, and they aged 20 to 70 years old. The consultation and answer part were additionally designed when collecting information by questionnaire survey among the patients to ensure the accuracy of data. A total of 321 experts from 28 provinces in China participated in the questionnaire survey. Most of the experts came from firstclass hospitals at grade 3. Doctors with professional title of attending physician and above were surveyed, and they came from the department of respiratory medicine, department of oncology, department of thoracic surgery, and department of radiotherapy. The experts with intermediate and deputy senior professional titles and above accounted for $76.64 \%$. $59.81 \%$ experts were directly involved in the treatment and diagnosis of COVID-19. These features of experts ensured the authoritativeness of the recommended therapeutic regimens.

COVID-19 is known for its extremely high infectivity, and patients with lung cancer are more susceptible to COVID-19 (3). Through the questionnaire survey, we found that the experts have a certain consensus on the treatment principle, the way of seeking medical treatment, the choice of hospital and medication scheme, and the recommendation of the preferred scheme. These findings are of high practical and clinical value. According to the current clinical guidelines, most of the experts recommend that anti-tumor therapy can be carried out as long as it is reasonable, although they preferred to wait for the end of the pandemic to weigh the trade-offs between efficacy and toxicity. Experts seem to have reached a consensus that in order to bring the best benefits to patients, we need to find a proper balance between efficacy and risk.

The changes of the therapeutic regimens for patients with advanced NSCLC during the pandemic are primarily based on expert recommendations. Therefore, it is highly crucial to establish a smooth doctor-patient communication channel during the pandemic, such as online video communications and cloud-based gratuitous treatment. Patients with advanced NSCLC sought medical care mainly through online public welfare consultations during the pandemic, and most of the patients' needs can be reasonably answered. The continuous improvement of China's communication network infrastructure and the rapid development of mobile Internet technology provide a strong guarantee for smooth doctor-patient communication. Chinese medical staff have been making selfless sacrifices and contribution to the fight against COVID-19, providing patient management and medical consultation services, and guiding the diagnosis and treatment and adverse events management for patients. Moreover, patients' trust in doctors and close collaboration between patients and doctors have created favorable conditions for the defeating COVID-19. However, due to the complexity of the diseases and some objective factors, including transportation and unbalanced regional development, the needs of some patients are still left unsatisfied, which is where more efforts and improvement are expected. While online medical consultation can solve some basic problems, complicated problems cannot be addressed unless through other 
measures.

Before and during the pandemic, local first-class hospitals at grade 3 remain the preferred choice for patients. It means that the first-class hospitals at grade 3 serve as a backbone force for safeguarding people's health. Before the pandemic, $75.12 \%$ of patients with advanced NSCLC chose to receive treatment at local and provincial first-class hospitals at grade 3 , where the treatment efficacy was better. However, the percentage of patients seeking medical care at nearby hospitals was increased from $17.45 \%$ before the pandemic to $35.02 \%$ during the pandemic. In the meantime, the percentage of patients seeking medical care at provincial renown first-class hospitals at grade 3 was decreased from $21.53 \%$ to $9.41 \%$ during the pandemic. Apparently, patients with advanced NSCLC were worried about contracting COVID-19 by traveling a long distance to seek medical care. Moreover, Chinese basic health care capability is being strengthened, and the first-class hospitals at grade 3 across China have played an important role in safeguarding people's health. Most of the patients' demands for primary health care can be met. At the same time, large renowned first-class hospitals at grade 3 have gradually become the treatment centers for difficult miscellaneous diseases and the clinical centers for making major technical breakthroughs. Furthermore, the patient is worried about contracting COVID-19 due to long-distance medical treatment, which further limits the patient's treatment options. Therefore, it is important to evaluate the benefitrisk ratio of patients with advanced NSCLC.

The early symptoms of COVID-19 include fever, dry coughs, and fatigue. Some patients are combined with nasal obstruction, running nose, pharyngalgia, myalgia, and diarrhea. These symptoms need to be distinguished from those of non-infectious diseases, such as vasculitis, dermatomyositis, and cryptogenic organizing pneumonia (4,5). Patients with advanced NSCLC receiving chemotherapy may also present with fatigue and pulmonary infection (6). The patients receiving targeted therapy may present with diarrhea, myalgia, fever, and interstitial pneumonia (7). Those receiving immunotherapy may present with fever, diarrhea, and immune-related pneumonia (8). For these reasons, the drug-related adverse events in patients with advanced NSCLC overlap with the symptoms of COVID-19, and differentiation is needed between the two to avoid mistaken diagnosis. The basis for differential diagnosis includes the history of exposure to the pandemic and contact with high-risk populations. Nucleic acid testing and radiographic examination can also be performed to distinguish.

Experts highly recommended targeted drugs for advanced NSCLC during the COVID-19 pandemic, and the oral anti-angiogenic drugs were the second most favorable. IPASS (9) provided convincing evidence for the use of gefitinib as the first-line treatment for patients with EGFR-mutated NSCLC. NEJ009 study further indicated that gefitinib plus pemetrexed and carboplatin significantly improved median progression-free survival (mPFS) than gefitinib monotherapy. We have noticed that the percentage of patients with performance status $(\mathrm{PS})=0$ (i.e., in good basic status) in the NEJ009 study was higher than that in other studies. Therefore, the overall survival (OS) was longer in these patients, indicating that the combination therapy may be more suitable for patients in good basic status. Recent years have witnessed the large-scale clinical use of third-generation EGFR-targeting drugs. FLAURA study (10) showed that osimertinib significantly improved mPFS and median OS (mOS) than the first-generation TKI (gefitinib/Tarceva). Moreover, the incidence of treatment-related adverse events of grade $\geq 3$ was lower with osimertinib ( $42 \%$ vs. $47 \%$ ), indicating a right balance between toxicity and OS. Furthermore, both NEJ026 (11) and RELAY studies (12) indicated that anti-angiogenic drugs combined with EGFR-TKIs could considerably improve PFS in patients with advanced NSCLC combined with controllable adverse events. Version 3.220 of the NCCN Guidelines (13) recommended anti-angiogenic drugs combined with EGFR-TKIs as the first-line therapy for EGFR mutation-positive NSCLC. Therefore, during the pandemic, the experts strongly recommended oral targeted drugs or targeted drugs combined with oral antiangiogenic drugs for the driver gene-positive patients with advanced NSCLC. By contrast, targeted therapy combined with chemotherapy was the less popular.

For the driver gene-positive or driver gene-negative patients with advanced NSCLC who were unable to return to hospitals for treatment and showed resistance to the TKI therapy, most of the experts recommended oral anti-angiogenic drugs as the home-based therapy. This therapeutic regimen could achieve a right balance between anti-tumor efficacy and COVID-19 risk. The ALTER 0303 trial (14) indicated that monotherapy with anlotinib hydrochlorideoral and anti-angiogenic drug could dramatically improve the PSF and OS as the third-line therapy and above for patients with NSCLC than placebo. The adverse events primarily included hypertension, fatigue, an increase of the thyroid stimulating hormone 
level, anorexia, hypertriglyceridemia and hand-foot syndrome. The anti-angiogenic drugs are easy to use and can control the adverse events. During the 2019 WCLC World Conference, Prof. Han from the Shanghai Chest Hospital Affiliated Shanghai Jiao Tong University School of Medicine reported the following outcome for the use of sintilimab combined with anlotinib as the first-line therapy for the advanced NSCLC: objective response rate (ORR) $72.7 \%$, disease control rate (DCR) $100 \%$, and incidence of grade $\geq 3$ adverse events $27.3 \%$ (15). These results indicated that anlotinib combined with other drugs as the first-line therapy could lead to satisfactory outcomes with controllable risk. During the pandemic, the oral multitarget anti-angiogenic drugs offered another treatment option for patients with advanced NSCLC.

Most of the experiments recommended drug discontinuation and quarantine for COVID-19 for patients with advanced NSCLC who contracted COVID-19 during chemotherapy or immunotherapy. In one study which recruited 915 patients with malignancies who received immunotherapy, 43 patients (5\%) had pneumonia, and the time of the onset of pneumonia varied from 9 days to 19.2 months (16). Radiographic examination is a very important diagnostic method for COVID-19. However, it is considered inadequate for the differentiation between immune-related pneumonia and COVID-19. For patients with negative driver gene and positive PD-L1 expression, the KEYNOTE-024 trial (8), Keynote-042 trial, and Keynote-189 trial showed that the immunotherapy with or without chemotherapy could significantly improve the survival of patients with advanced lung cancer. According to our survey, the experts strongly recommended immunotherapy with or without chemotherapy. During the pandemic, the experts highlighted the importance of personal protection. Extra attention should be given to the adverse events caused by the immune checkpoint inhibitors, such as fever, diarrhea, and immune-related pneumonia (8) and chemotherapy-related toxic and side effects (6). Immunotherapy is associated with distinctive side effects, which need to be carefully observed to achieve early discovery, early diagnosis and early treatment.

Our study also had certain conspicuous limitations. Firstly, the survey sample size was not sufficiently large, which might impair the convincingness of the conclusions. Secondly, the questions contained in the questionnaire were limited and did not cover every aspect of advanced NSCLC. Thirdly, the questionnaire responses were the only source of data, which led to population selection bias. Since the survey findings were not fully supported by the objective evidence in evidence-based medicine, they only serve as a reference for patients with advanced NSCLC during the COVID-19 pandemic.

The diagnosis and treatment of lung cancer are challenged during the COVID-19 pandemic. During the COVID-19 pandemic, although the diagnosis and treatment of lung cancer are affected to varying degrees, most of patients with lung cancer in China are still able to receive timely diagnosis and treatment either by online public welfare consultation or at nearby hospitals. For drug selection, immunotherapy and chemotherapy should be administered with caution to patients with NSCLC. According to the questionnaire survey, targeted therapy and oral anti-angiogenic drugs were the most commonly recommended by experts. This recommendation also agreed with the actual choice made by the general patient population. Personal protection should be strengthened for patients with NSCLC who are needed for hospitalization to get immunotherapy and chemotherapy. Effective communication mechanisms should be established with the attending physician to cope with potential drug-related adverse events. A right balance between the benefits and risks of the anti-tumor treatment is particularly vital under this unique situation to achieve maximum benefits for the patients with NSCLC. Based on the above consideration, most experts strongly recommended the targeted therapy and oral anti-angiogenic drugs. Our results agreed with the actual choice made by the advanced NSCLC patients.

\section{Acknowledgments}

Funding: This study was funded by the Science and Technology Program of Guangzhou, China (No. 202008010006), Guangdong Province Science and Technology Innovation Strategy Special Fund of Guangdong Province (No. 2020A1111350025).

\section{Footnote}

Reporting Checklist: The authors have completed the SURGE reporting checklist. Available at https://apm. amegroups.com/article/view/10.21037/apm-21-72/rc

Data Sharing Statement: Available at https://apm.amegroups. com/article/view/10.21037/apm-21-72/dss 
Conflicts of Interest: All authors have completed the ICMJE uniform disclosure form (available at https://apm. amegroups.com/article/view/10.21037/apm-21-72/coif). The authors have no conflicts of interest to declare.

Ethical Statement: The authors are accountable for all aspects of the work in ensuring that questions related to the accuracy or integrity of any part of the work are appropriately investigated and resolved. The study was conducted in accordance with the Declaration of Helsinki (as revised in 2013). The study was approved by the ethics committee of the First Affiliated Hospital of Guangzhou Medical University (Ethics Number: 2020 No. 35). All subjects signed informed consent.

Open Access Statement: This is an Open Access article distributed in accordance with the Creative Commons Attribution-NonCommercial-NoDerivs 4.0 International License (CC BY-NC-ND 4.0), which permits the noncommercial replication and distribution of the article with the strict proviso that no changes or edits are made and the original work is properly cited (including links to both the formal publication through the relevant DOI and the license). See: https://creativecommons.org/licenses/by-nc-nd/4.0/.

\section{References}

1. Chan JF, Yuan S, Kok KH, et al. A familial cluster of pneumonia associated with the 2019 novel coronavirus indicating person-to-person transmission: a study of a family cluster. Lancet 2020;395:514-23.

2. Epidemiology Working Group for NCIP Epidemic Response, Chinese Center for Disease Control and Prevention. The epidemiological characteristics of an outbreak of 2019 novel coronavirus diseases (COVID-19) in China. Zhonghua Liu Xing Bing Xue Za Zhi 2020;41:145-51.

3. Liang W, Guan W, Chen R, et al. Cancer patients in SARS-CoV-2 infection: a nationwide analysis in China. Lancet Oncol 2020;21:335-7.

4. Lin L, Li TS. Interpretation of "Guidelines for the Diagnosis and Treatment of Novel Coronavirus (2019$\mathrm{nCoV}$ ) Infection by the National Health Commission (Trial Version 5)". Zhonghua Yi Xue Za Zhi 2020;100:E001.

5. General Office of National Health Commission, General Office of National Administration of Traditional Chinese
Medicine. Diagnosis and treatment plan of Novel Coronavirus Pneumonia (trial version sixth). [2020-02-29]. Available online: http://www.nhc.gov.cn/xcs/zhengcwj/202 002/8334a8326dd94d329df351d7da8aefc2.shtml

6. Scagliotti GV, Parikh P, von Pawel J, et al. Phase III study comparing cisplatin plus gemcitabine with cisplatin plus pemetrexed in chemotherapy-naive patients with advanced-stage non-small-cell lung cancer. J Clin Oncol 2008;26:3543-51.

7. Mok TS, Wu YL, Thongprasert S, et al. Gefitinib or carboplatin-paclitaxel in pulmonary adenocarcinoma. $\mathrm{N}$ Engl J Med 2009;361:947-57.

8. Reck M, Rodríguez-Abreu D, Robinson AG, et al. Updated analysis of KEYNOTE-024: pembrolizumab versus platinum-based chemotherapy for advanced nonsmall-cell lung cancer with PD-L1 tumor proportion score of 50\% or greater. J Clin Oncol 2019;37:537-46.

9. Hosomi Y, Morita S, Sugawara S, et al. Gefitinib alone versus gefitinib plus chemotherapy for non-small-cell lung cancer with mutated epidermal growth factor receptor: NEJ009 study. J Clin Oncol 2020;38:115-23.

10. Ramalingam SS, Vansteenkiste J, Planchard D, et al. Overall survival with osimertinib in untreated, EGFR-mutated advanced NSCLC. N Engl J Med 2020;382:41-50.

11. Saito H, Fukuhara T, Furuya N, et al. Erlotinib plus bevacizumab versus erlotinib alone in patients with EGFR-positive advanced non-squamous non-small-cell lung cancer (NEJ026): interim analysis of an open-label, randomised, multicentre, phase 3 trial. Lancet Oncol 2019;20:625-35.

12. Nakagawa K, Garon EB, Seto T, et al. Ramucirumab plus erlotinib in patients with untreated, EGFRmutated, advanced non-small-cell lung cancer (RELAY): a randomised, double-blind, placebo-controlled, phase 3 trial. Lancet Oncol 2019;20:1655-69.

13. National Comprehensive Cancer Network. NCCN clinical practice guidelines in oncology: non-small cell lung cancer (2020.V3). [2020-02-29]. Available online: http:// www.nccnchina.org/nccn-guidelines-china.aspx

14. Han B, Li K, Wang Q, et al. Effect of anlotinib as a thirdline or further treatment on overall survival of patients with advanced non-small cell lung cancer: the ALTER 0303 phase 3 randomized clinical trial. JAMA Oncol 2018;4:1569-75.

15. Han BH, Chu TQ, Zhong RB, et al. JCSE01.11 Efficacy and safety of sintilimab with anlotinib as first-line therapy 
for advanced non-small cell lung cancer (NSCLC). J Thorac Oncol 2019;14:S129.

16. Naidoo J, Wang X, Woo KM, et al. Pneumonitis in patients treated with anti-programmed death-1/ programmed death ligand 1 therapy. J Clin Oncol 2017;35:709-17.

Cite this article as: Liu M, Zhao W, Li S, Chen L, Zhou C. Current status of diagnosis and treatment of advanced nonsmall cell lung cancer in China during the COVID-19 pandemic. Ann Palliat Med 2022;11(4):1231-1240. doi: 10.21037/ apm-21-72 